\title{
Plasma physics for fusion reactor system codes: framework and model code
}

\author{
E. Fable, C. Angioni, M. Siccinio, H. Zohm \\ Max-Planck-Institut für Plasmaphysik, 85748 Garching, Germany \\ E-mail: emiliano.fable@ipp.mpg.de
}

\begin{abstract}
Design of a fusion reactor power plant requires putting together physics elements (plasma physics) and engineering elements (plant characteristics). System codes are tools that perform integrated plant design taking into account those two aspects. Design optimization can also be carried out, to produce the "best" reactor design, according to some pre-defined figures of merit. However, presently used system codes often lack a plasma model with sufficient level of realism in terms of description of plasma processes and non-linearities. In this work, for the first time, a framework in which the plasma model should interact with the engineering elements is detailed, giving the attention on the logic of how the plasma physics should be represented inside the system codes. Ultimately, no ambiguity is left on how the problem should be addressed and solved. Concrete details on how the plasma model should be written are also presented. A novel code PLASMOD has been written which incorporates these elements and can be used in a generic engineering system code.
\end{abstract}




\section{Introduction}

Fusion reactors are foreseen as the main source to replace coal, fission -based power plants for the future needs of mankind. Comprehensive designs of such a reactor have already been undertaken worldwide. In Europe particular focus is now devoted to the tokamak DEMO design $[1,2]$. To design such a complex machine, many elements have to be put together including, in particular: the plasma, the magnets, the blanket, the coils. Each element has its own characteristics, constraints, limits, and requirements. The available engineering knowledge that goes into those sub-systems is put together into System Codes (SC), comprehensive software tools that aim at providing a complete design of the machine, including costs. The SC is often equipped with an optimiziation routine that also looks for the best design, in terms of some predefined figure of merit. One of the most used SC in EUROFusion is PROCESS [3], which contains all such elements defined above. Example of other codes which are in development or are used elsewhere are SYCOMORE [4], NOVA/Blueprint [5], MIRA [6].

SC usually focus on the engineering aspects, materials, magnets, force limits, irradiation limits, etc., and the plasma itself which provides the fusion power is simply described as a 0D entity via some widely used scaling laws (e.g. the ITER Physics Basis 1998 (IPB98) scaling law for the confinement time [7]) and prescribed profiles. Such a plasma description provides already a rough estimate of the fusion power produced by the plasma, and its dependence on some global parameters. However, many drawbacks render this approach practically useless to define what would be the best design and its actual performance, for the following reasons:

1) Profile effects are very important in determining the actual fusion power produced at constant $\mathrm{H}$ factor [8]. Parametrized profiles are already in used in system codes, however it is known from experimental observations that profile shapes are not self-similar (i.e. the coefficients of the parametrization are actually parameterdependent), in particular the relation between density and temperature is complex and cannot be captured by a simple parametrization;

2) The physics of core and pedestal in an $\mathrm{H}$-mode plasma are completely different and cannot be "independently" captured by a single global scaling;

3) Divertor protection requirements cannot be easily computed in a OD setting;

4) Prediction of the bootstrap current (as well as of the plasma equilibrium) requires knowledge of realistic plasma profiles;

5) Many non-linear processes happening in the plasma are local in radius, and thus cannot be included in a 0D framework;

Since including, at least, a 1D description of the plasma would not dramatically require more computational time for the $\mathrm{SC}$, it is then preferrable to describe the plasma with profile effects and with relevant physics so that the inter-dependencies and non-linearities that dominate the plasma behavior, as both described theoretically and observed experimentally, are retained.

The paper is organized as follows: section 2 describes the physics interfaces between 
plasma and technology. Section 3 discusses the relevant plasma processes that should go into the plasma model. Section 4 describes in detail the coupling scheme taking into account the previous sections results. Section 5 describes the new code PLASMOD and shows a few examples of results obtained with the code. Section 6 discusses some technical issues. Section 7 draws the conclusions.

\section{Plasma-techonology interfaces}

The first realization of how a plasma model should couple to the engineering modules, comes directly from the natural way of interaction between the plasma and the outside materials.

Assuming that the plasma is confined inside the first-wall (plasma includes both core and the scrape-off-layer/divertor region), than the plasma interacts in the following ways:

1) The burning plasma core produces neutrons and line/synchrotron/bremsstrahlung radiation (both volumetric reactions), these impact the first wall elements (blanket, limiters, shielding elements, central solenoid column, etc.);

2) The heat and particle fluxes outside of the plasma separatrix through the scrapeoff-layer (SOL) are convected/conducted directly to the first wall (e.g. perpendicular transport) and to the divertor tiles (parallel transport);

3) Plasma particle content (i.e. inventory) is provided by fueling/pumping and wall recycling. E.g. since the density peaking impacts the choice of the pedestal top density (if one wants to have a specific line average density), profile effects are important to estimate the actual amount of required fueling;

4) The plasma is controlled both magnetically (external coils) and kinetically (auxiliary heating). As an example, tailoring of the safety factor, which could both increase the bootstrap current, enhance confinement, and lead to a steady-state inductiveless scenario, can only be obtained when profiles are computed, as the position where current drive is applied impacts all these elements;

After having identified these interfaces, it will be then straightforward to write down the exchange parameters in I/O from plasma to technology and viceversa.

\section{Plasma processes and plasma model}

The plasma could be divided in 4 main regions: the plasma core (up to pedestal top), the pedestal up to the separatrix, the SOL, and the divertor region. In figure 1 these regions are displayed, where core (1) energy is transport through the pedestal to the SOL (2), and then particle end energy flows to the divertor (3). In each region, physics processes develop which are characterized by specific parameters (that can also be cast in dimensionless form) and non-linear phenomena (that is, they do not follow simple linear monotonic behavior with respect to control parameters). 


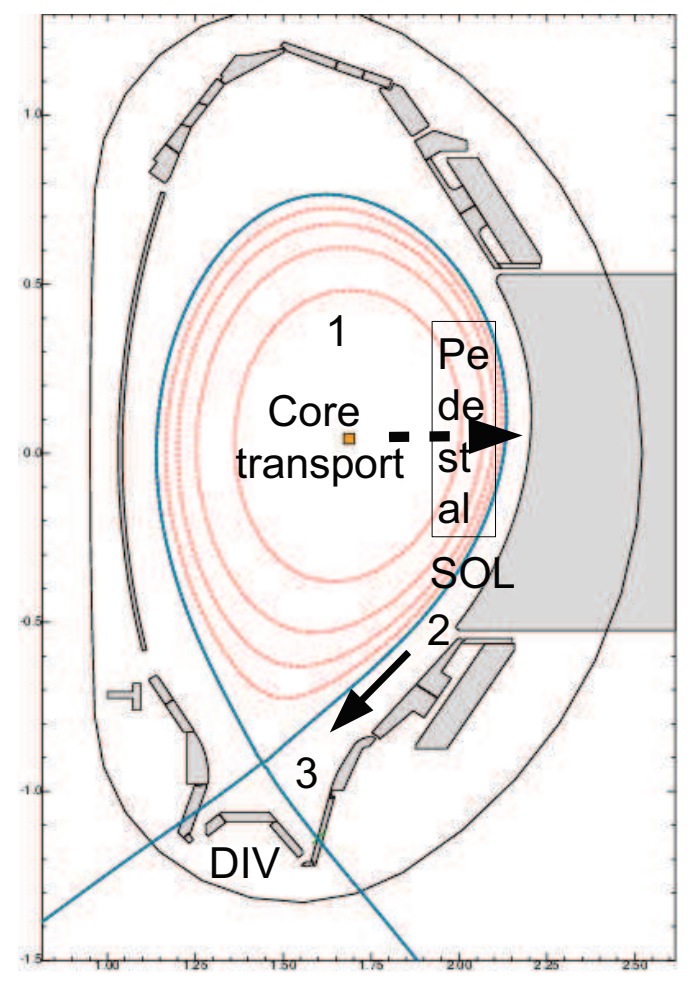

Figure 1. Regions of plasma processes. 1) Core + pedestal, 2) SOL, 3) divertor.

\subsection{Core plasma}

The core plasma is heated by auxiliary heating, and by the fusion reactions themselves. Since relevant fueling is mostly done in the edge region, the core density profile is basically determined by a balance of diffusion and convection. Once the sources are defined, together with the plasma geometrical parameters (shaping, field, current), plasma confinement can be computed assuming for example turbulence, neoclassical and some form of MHD transport. The pedestal top would act here as a boundary condition for the core plasma model.

The core confinement is determined by multi-scale processes: Larmor-scale turbulence, collisional (neoclassical) transport, and MHD (sawteeth, NTMs, ...). Each process can in principle be modeled given the local inputs in terms of profiles (geometry, temperatures, particle densities, current density). Just to make an example, referring to typical diffusion rates, collisional transport would scale as $D_{\text {coll }} \sim A^{2} q^{2} \rho_{L}^{2} \nu \mathrm{m}^{2} / \mathrm{s}$, where $A, q$ are respectively the aspect ratio and the safety factor, while turbulent transport as $D_{\text {turb }} \sim\left(\rho_{L} / R\right)^{2} c_{\mathrm{S}} R$, where $\rho_{L}, c_{\mathrm{s}}, R$ are respectively the Larmor radius, the sound speed, and the major radius. These basic diffusion rates already show the non-linear dependencies with respect to the local temperature.

Impurities coming from the edge would also be present in the core, producing radiation, dilution and effecting plasma confinement. 


\subsection{Pedestal}

Plasma regimes are often divided in low-confinement, or "L-mode", and highconfinement, or "H-mode", with addition of "improved" regimes that can also be part of the core, but often are edge phenomena (improved L-modes, I-modes). In any case, physics of the edge layer (few cms inside the separatrix) is very different from core physics. That is why the pedestal region is treated on a different footing.

The plasma model should recognize in which regime it is given the local or the global parameters (e.g. $\left.P_{\mathrm{sep}} / P_{\mathrm{LH}}\right)$ and consequently decide what to do with the pedestal.

Let one suppose that it is an $\mathrm{H}$-mode regime. In this case, the pedestal height and width could be modeled assuming type 1 ELMy regime, and employing computational tools that combine pedestal MHD and kinetic stability like EPED [9]. Notice that, in reality, the plasma regime could also be different, for example type 3 ELMy, or ELMmitigated or suppressed via application of RMPs $[10,11]$. However, for the latter regimes a systematic study to extract parametric dependencies in a robust way is still ongoing and no definite scalings can be used in the presented code.

In L-mode, the pedestal would not exist as such but the core would be continued up to the separatrix.

\subsection{SOL/divertor}

The SOL and divertor regions can be described as both determining parallel transport to the plates, and perpendicular transport to the first wall. Many codes are available that solve this problem in a rather complete way, up to including neutrals for example [12]. However, in this context one cannot afford to routinely run such codes as the computational costs are too high. In practice, 0D recipes could be used (e.g. the two-point model), or one could use routines computing 1D radiation profiles and the consequent heating at the divertor from upstream.

\section{System codes coupling logic including the plasma model}

Now that all the pieces are set for the coupling, the latter can be performed. It is assumed that the System Code consists of a main "optimizer" (or say, a main place where gross engineering parameters are defined). This will provide the following parameters:

- to plasma model (INPUT): major radius $\mathrm{R}$, reference field $B_{\mathrm{T}}$, plasma shape parameters (e.g. for a three-moment-type description: $k, \delta, a$ ), plasma safety factor $q_{95}$. It would also tell the plasma which constraints to satisfy: loop Voltage must be lower than $x \rightarrow$ requires auxiliary current drive; divertor heat loads / temperature must be lower than $x \rightarrow$ requires impurity seeding; plasma density must be $x \rightarrow$ requires fueling; $P_{\text {sep }} / P_{\text {LH }}$ must be between $x_{1}-x_{2} \rightarrow$ requires either impurity puffing or auxiliary heating (alternatively, $P_{\text {fus }}$ itself could be given as additional requirement), fuel mix (D-T ratio).

Once the plasma model has run, it produces a set of parameters and requirements to be delivered back to the technology side: 
- from plasma to technology (OUTPUT): required $P_{\text {aux,CD }}$ for current drive; required $P_{\text {aux,heat }}$ for heating; required impurity seeding for core and for divertor; required fueling in terms of required $\mathrm{D}, \mathrm{T}$ influx into the plasma in $\mathrm{p} / \mathrm{s}$; plasma profiles: temperatures, densities, current density, radiation source, neutron source; divertor heat flux and divertor temperature; global parameters: fusion power, normalized pressure $\beta_{\mathrm{N}}$, plasma current, type of regime, confinement time $\tau, \ldots$

\subsection{Plasma model: core/pedestal transport}

Core transport processes (turbulence, collisions, ideal and resistive MHD modes) determine the steady-state temperature and density profiles from magnetic axis up to pedestal top position. In this region is commonly assumed that the transport mechanisms are dominantly turbulence of ITG/TEM/ETG type and MHD phenomena as sawteeth and NTMs $[13,14,15,16]$. Regarding the current profile, this is usually assumed to be dictated by neoclassical resistivity [17]. The bootstrap current can also be readily computed [17].

The profiles can then be solved using standard 1D transport equations as in stateof-the-art transport codes $[18,19,20,21]$. These equations are solved strictly for steady-state, i.e. without time derivatives. The solution can be obtained using standard Newton-schemes, modified to account for the non-linear transport coefficients (as an example, e.g. [22, 23]).

The pedestal acts as the boundary condition for the core profiles. The pedestal height and width can be assigned via the pertinent physics processes. As an example, in this recent work [24] a pedestal scaling provided by a set of EPED runs specifically devised around DEMO parameters was used.

\subsection{Plasma model: SOL/divertor transport}

The same model used in [24] could be used here, or alternatively something as in [25]. In practice what the model does is taking in input the power crossing the separatrix, the separatrix density, and computes the divertor power load, with assumptions on the heat flux expansion (e.g. from geometrical considerations: a flux tube starting at the midplane with an area $A_{\text {up }}$ will impact on the divertor with an area $A_{\text {div }}$ which ratio $A_{\text {div }} / A_{\text {up }}$ depends on magnetic equilibrium and surface inclination), and the SOL/divertor parallel and perpendicular transport.

\subsection{Plasma model: global particle balance}

To link the plasma density to the fueling and pumping, which will impact the machine design, one should provide also a global particle balance model. Such a model can be either described as a multi-region model, i.e. plasma core (inside separatrix), SOL region, and divertor region, where particle confinement times in the different regions are 
assigned, and would link fueling and density in the following way:

$$
\frac{d N_{j}}{d t}+\Sigma_{i} D_{i j}\left(N_{j}-N_{i}\right)=S_{j}-P_{j}
$$

where $j$ is "core", "SOL", "divertor", $N_{j}$ is the particle content in region "j", $S_{j}$ is the source, and $P_{j}$ is the sink. At steady-state $P_{j}=S_{j}$, and the global particle confinement time is given by $\Sigma_{j} N_{j} / \Sigma_{j} S_{j}=\Sigma_{j} N_{j} / \Sigma_{j} P_{j}$. Since $P_{j}$ can be described as $P_{j}=N_{j} / \tau_{j}$, with $\tau_{j}$ the confinement time of particles in region $j$, the global confinement time would then be used as input to calibrate the $\tau_{j}$. As an example, for He, the global confinement time is usually described as a ratio of the core energy confinement time, i.e. $\tau_{\mathrm{He}} \approx(\ldots) \tau_{\mathrm{E}}$. This ratio is often given as having an experimentally observed lower limit of $\sim 5[26,27$, which also is estimated as a maximum limit to avoid losing reactor performance [28]. Notice that the value for He reflects the effect of recycling and pumping on He global confinement.

In practice, what is done in the present PLASMOD code, the fueling requirement is computed by dividing the total amount of particles in the core, for each species, and dividing it by the global confinement factor. This procedure makes sense for D, T, Xe, Ar, and in case of considerations about the pumping, for He.

\subsection{Plasma model: magnetic equilibrium}

Magnetic equilibrium can be readily solved using the Grad-Shafranov equation, provided the shape of the plasma boundary and the pressure and current profile inside the plasma. As such, the coupling between core transport and equilibrium can be achieved robustly inside the plasma model. Once a fully self-consistent solution is obtained, the plasma current and pressure, together with the $2 \mathrm{D}$ equilibrium, can be passed back to the engineering side of the SC for modeling of the coils and for global stability calculations. In case the plasma boundary shape needs to be changed, it is then easy to rerun the plasma model with the new shape, up to convergence.

\section{The plasma code PLASMOD}

A new software tool has been written, which is called "PLASMOD" (stands for PLASma MODel), which solves 1D transport equations inside the plasma core, coupled to a Grad-Shafranov solver for the 2D plasma equilibrium inside a specified shape. The plasma model equations include power and particle balance, plus ad-hoc models for SOL transport and divertor heat loads calculations.

At present the employed Grad-Shafranov solver is the EMEQ solver [29], which is also routinely used in ASTRA.

\subsection{Example of results}

As a test case it is employed the EU-DEMO 2015 scenario [2], which global parameters are reported here: 


\begin{tabular}{|c|c|}
\hline DEMO parameter & Value \\
\hline $\mathrm{A}$ & 3.1 \\
\hline $\mathrm{R}$ & 9.07 \\
\hline$B_{\mathrm{T}}$ & 5.67 \\
\hline $\mathrm{k}$ & 1.75 \\
\hline$\delta$ & 0.38 \\
\hline$q_{95}$ & 3.5 \\
\hline
\end{tabular}

Table 1. EU-DEMO 2015 (DEMO) global parameters.

- $\mathrm{A}=3.1 ;$ aspect ratio

- $\mathrm{R}=9.07 \mathrm{~m}$; major radius

- $B_{\mathrm{T}}=5.67 \mathrm{~T}$; magnetic field

- $\mathrm{k}=1.75$; edge elongation

$-\delta=0.38$; edge triangularity

- $q_{95}=3.5$; safety factor $\rightarrow$ this results in a plasma current of 18.5 MA

Aside from these global parameters, other physics parameters are assigned as:

- $n_{\text {pedtop }} / n_{\mathrm{G}}=0.9$; ratio of pedestal top to Greenwald density

- fuel mix is $0.5 \mathrm{D}$ and $0.5 \mathrm{~T}$ per unit fuel

- $\tau_{\mathrm{He}, \mathrm{p}} / \tau_{\mathrm{E}}$, ratio of He particle to plasma energy confinement times, is fixed at 5 . This is used to compute the He concentration selfconsistently as explained in previous sections, whereas the He source is simply the fusion power divided by alpha particle energy (all alphas become thermalized eventually before escaping).

As for the constraints:

- $P_{\text {sep }} / P_{\mathrm{LH}}$, i.e. ratio of separatrix to $\mathrm{L}-\mathrm{H}$ transition power (Martins scaling is employed here [30]) must be between 1.1 and 1.2. Notice that the original Martins scaling is given for the separatrix power accounting for auxiliary heating, but not for radiation. The reason why here $P_{\text {sep }}$ is used, which is including the loss due to radiation, is because it is expected that radiation, while impacting only electrons, will actually also impact ions due to the equipartition power which is shorter as the confinement time in a machine such as DEMO (also in ITER).

The employed transport model is a simple gyro-Bohm scaling with some radial profile for the transport coefficient that would yield a realistic shape of the temperature profiles. The particle convection, directed inward, is sized as to reproduce expectations from theoretical modeling [31, 13]. For this specific case, it predicts a confinement quality factor of $H \approx 0.95$. Notice that the pedestal height is determined using a scaling provided by [32], which is based on the physics-based EPED model [9].

A discussion about the possible approach to compute core confinement is now in order. In principle 3 approaches are possible: 1) the global confinement is assigned via the $\mathrm{H}$ factor, as well as the pedestal height is assigned (maybe scanned); 2) the global confinement is assigned via the $\mathrm{H}$ factor, and the pedestal height is assigned via 

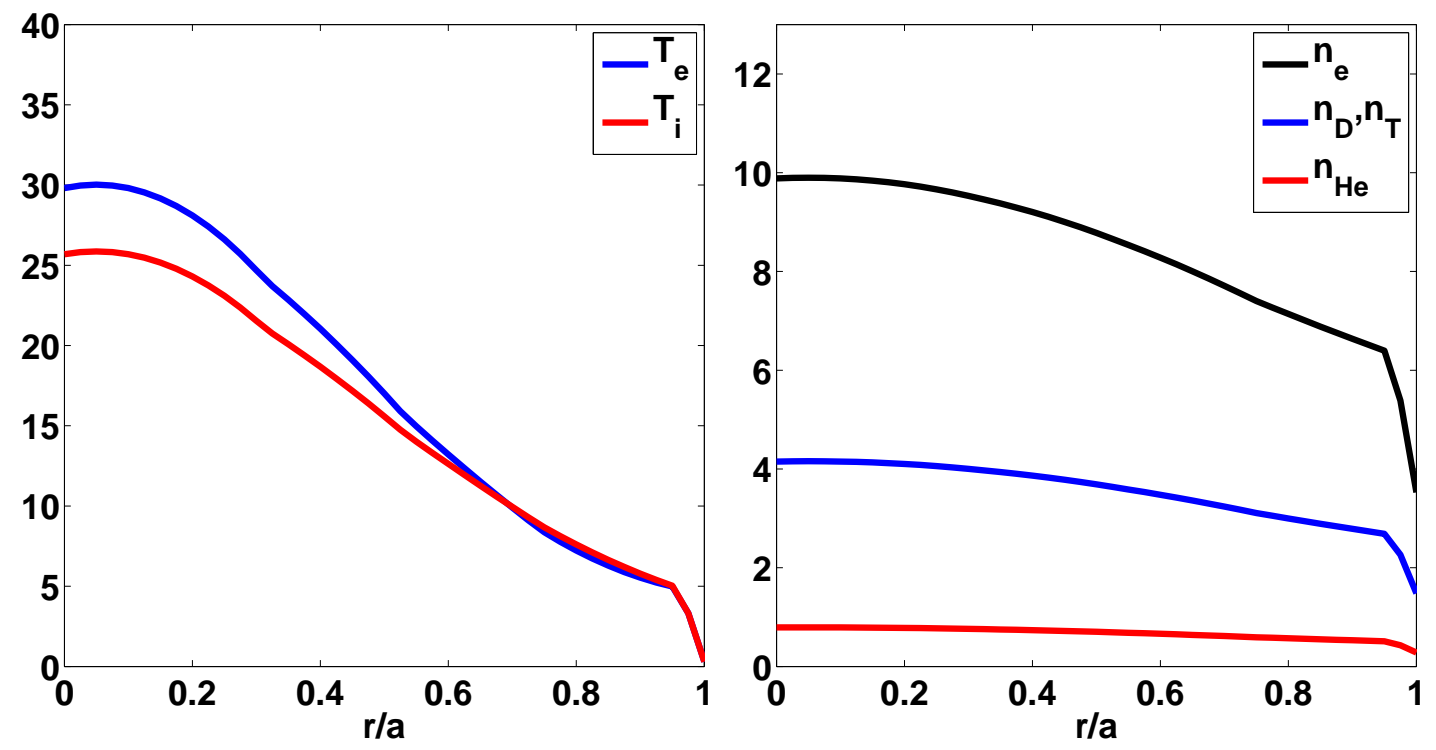

Figure 2. a) electron and ion temperature profiles $T_{\mathrm{e}}, T_{\mathrm{i}}$ in $\mathrm{keV}$; b) Electron, deuterium/tritium, He density profiles $n_{\mathrm{e}}, n_{\mathrm{D}}, n_{\mathrm{He}}$ in $10^{19} \mathrm{~m}^{-3}$. These profiles are obtained for the global parameters displayed in table 1.

a scaling; 3) global confinement is an output of the given model for core transport and the scaling for the pedestal height (this is the approach presently used here).

Approach 3) is of course the preferred one to have predictive capability without relying on the global confinement database which does not distinguish between core and pedestal. Nonetheless, approach 2) still makes sense since it links global confinement scaling to partly theory-based (or also empirically-based) scaling for the pedestal, and the resultant core part. Approach 1) instead can be misleading since, depending on the actual value of the pedestal height, can lead to completely arbitrary values of core temperatures, and ultimately fusion power. That is why it is suggested to rely on approach 2) and 3) if possible, and avoid approach 1).

The obtained steady-state profiles of temperatures and densities are shown in figure 2, while the current density and bootstrap current profiles are shown in figure 3 . Notice that the safety factor profile $q$ has a flattening in the plasma core which clamps it at $q=1$ since our tool also employs a continous sawtooth model to prevent it falling below 1 (which would lead to an ideal kink at some point).

The global parameters obtained for this specific case are:

- $H, \tau_{\mathrm{E}}=0.95,3.5 \mathrm{~s}$

- $P_{\text {fus }}=1.53 \mathrm{GW}$

- $I_{\mathrm{p}}=18.6 \mathrm{MA}$

- $V_{\text {loop }}=0.025 \mathrm{~V}$

- $P_{\text {sep }, \mathrm{LH}}=133,111 \mathrm{MW}$

$-P_{\text {aux }}=0$.

$-c_{\mathrm{Xe}, \mathrm{He}}=0.00015,0.088$ 

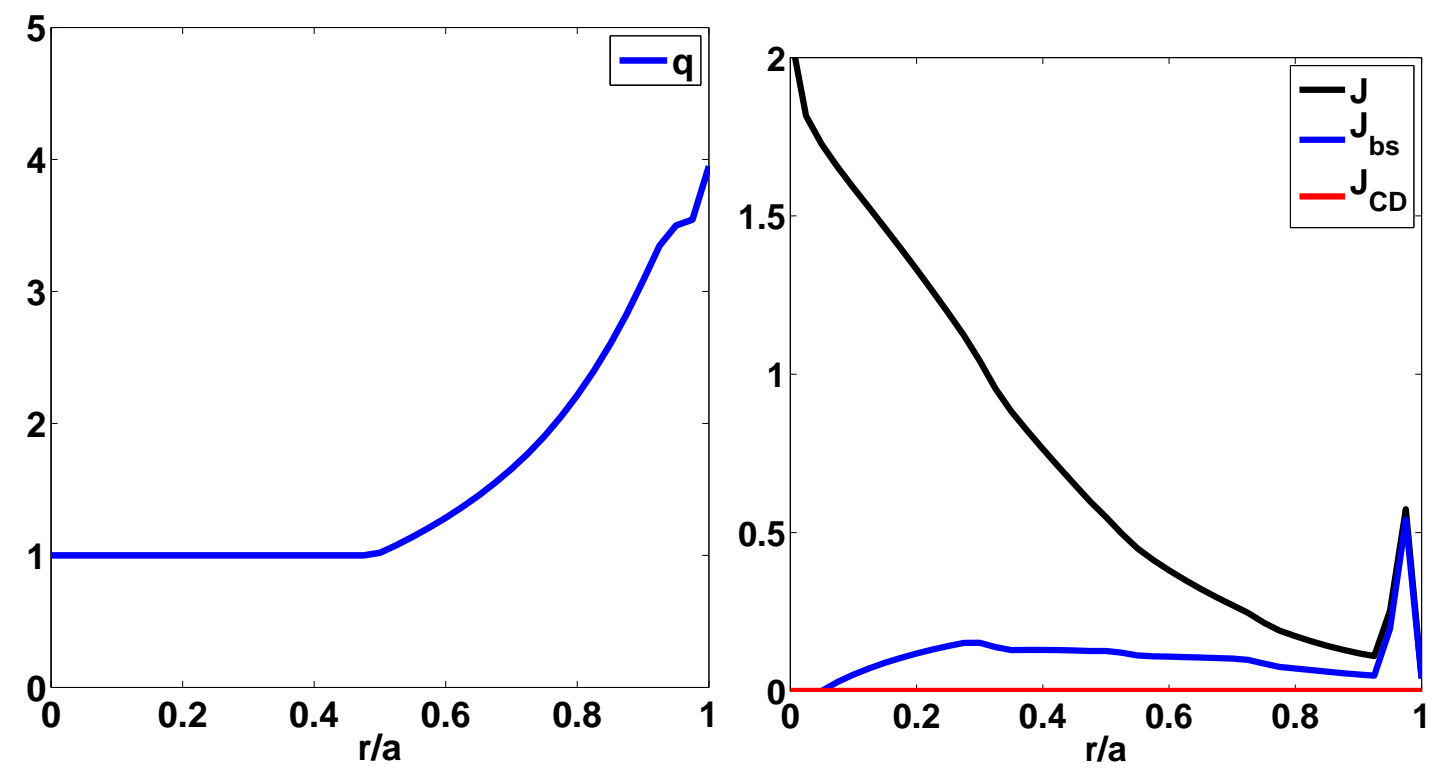

Figure 3. a) safety factor profile $q$; b) plasma current density profile in $M A / \mathrm{m}^{2}$, bootstrap and current driven currents $J_{\mathrm{bs}}, J_{\mathrm{CD}}$ (same units).

Note that the radiation model used for computing line radiation from Xe comes from [33].

As it can be seen, all the requirements are reached, and the results are consistent with them, with the magnetic equilibrium, and with the prescribed physics transport processes.

To have confidence in these results, the output of PLASMOD are then used into ASTRA run in interpretative mode. It is checked that the power balance diffusivities coincide indeed with the ones obtained in PLASMOD.

\section{Technical issues related to control}

In the previous section, upon describing the way the plasma model is coupled to the technology modules, one can see that estimates of required auxiliary power and fueling can be readily given, at least for steady-state operation (in the sense of quiescent plasma without perturbations). In a SC setting, these can then be compared to limits and technological constraints. In the present context, this is not yet achieved (coupling to an actual SC is in progress and will be presented in a future publication).

If magnetic equilibrium has to be addressed on the technology side (which makes more sense since it will define the PF, CS and TF coils), the plasma model readily delivers the $(R, Z)$ map of the plasma current density, which can be given to a freeboundary Grad-Shafranov solver for the coils design.

Now this would completely close the problem at least for the flattop phase of the plasma. However, the problem of dealing with current ramp phases and with transient 
phenomena itself has not been addressed. In present system codes, the current ramps are already included in some simplified way. At this point, it is not the scope of this paper to go into the details of the current ramp design, however what is usually assumed is that the most stringent requirement in the current ramps, apart from magnetic control, is that the installed power is sufficient to enter into $\mathrm{H}$-mode at some point during the ramp-up or as soon as the flattop is reached. What instead is not usually considered is the problem of transients.

Magnetic control for the vertical instability could be assessed by considering the safety margin against the VDE (vertical displacement event) which can be computed based on the instantaneous equilibrium, the field map, and the wall parameters (resistance and inductance).

For kinetic control, the situation becomes even more complicated since the plasma can develop a whole range of instabilities. The requirements on the installed power are then dependent on the strategy choosen to deal with these instabilities (e.g. sawteeth, NTM), and so require refined calculations which may in the future be possible to include in the plasma model. Nonetheless, one could try to estimate the required installed power for some of the most expected large instabilities. Just to make an example, one could expect that, if the pedestal temperature drops by a certain percentage of the initial temperature, this will require some power to avoid radiation collapse (or if there is a transient increase in radiation due to some external sources). Building up a matrix of "required power versus type of event" could become an automathized process inside the plasma model in the future.

\section{Conclusions}

In this work, a specific framework for the integration of a complete plasma model in reactor design system codes is given.

The coupling logic is based on a more sophisticated way in which the plasma interacts with the surrounding structures, together with the way in which the plasma would be tailored to satisfy engineering and basic discharge requirements.

The presented framework can readily be implemented in existing system codes and will allow one to avoid inconsistencies between plasma physics and technological design, plus when more realistic physics models for the different plasma processes will be available, their inclusion will not alter anything in the structure of the system code.

The presented framework displays clearly what are inputs and what are outputs for/from the plasma model to establish a logical dialogue with the techonology modules.

A novel code, called PLASMOD, has been written to perform the aforementioned task, and can be readily coupled to a generic systems code for fusion power plant studies. Future developments of the code, which are readily available for implementation, are: inclusion of several heating/current drive schemes in terms of deposition location choice and calculation of split between electron and ion heating (e.g. from NBI), allow for more choices of core and pedestal transport models/scalings, allow for more choices 
of simplified SOL/divertor models, improve the core transport model to follow stateof-the-art theoretical results. Regarding heating and current drive schemes, despite the auxiliary heating being usually sub-dominant to the alpha heating (except in fully non-inductive scenarios), it would allow to perform studies with q-profile tailoring to optimize performance and stability.

\section{Acknowledgments}

The authors are grateful to Dr. H. Lux, M. Coleman, F. Franza, R. Kemp, M. Kovari for useful discussions on system codes. This work has been carried out within the framework of the EUROfusion Consortium and has received funding from the Euratom research and training programme 2014-2018 under grant agreement No 633053. The views and opinions expressed herein do not necessarily reflect those of the European Commission.

\section{References}

[1] R. Wenninger et al 2014 Nucl. Fusion 54114003

[2] R. Wenninger et al 2015 Nucl. Fusion 55063003

[3] M. Kovari et al. Fusion Engineering and Design Volume 89, Issue 12, December 2014, Pages 30543069

[4] C. Reux et al. 2015 Nucl. Fusion 55073011

[5] M. Coleman et al., Building an in-silico tokamak: integrated multi-physics modelling for fusion engineering, presented at SOFE-27, Shanghai, China, 2017

[6] F. Franza et al., Fusion Engineering and Design, 98-99:1767-770, 2015

[7] V Mukhovatov et al 2003 Plasma Phys. Control. Fusion 45 A235

[8] G. Giruzzi et al 2015 Nucl. Fusion 55073002

[9] P.B. Snyder et al 2011 Nucl. Fusion 51103016

[10] R. A. Moyer et al., Physics of Plasmas 24, 102501 (2017)

[11] Yueqiang Liu et al 2016 Plasma Phys. Control. Fusion 58114005

[12] A S Kukushkin and H D Pacher 2002 Plasma Phys. Control. Fusion 44931

[13] E. Fable et al 2017 Nucl. Fusion 57022015

[14] J. Y. Kim, and H. S. Han, Physics of Plasmas 24, 072501 (2017);

[15] I.T. Chapman et al 2012 Nucl. Fusion 52063006

[16] E. Poli et al 2015 Nucl. Fusion 55013023

[17] O. Sauter et al., Physics of Plasmas 6, 2834 (1999)

[18] G. V. Pereverzev et al., IPP Report 5/42 (August 1991)

[19] E. Fable et al., Plasma Phys. Control. Fusion 55, 124028 (2013)

[20] G Genacchi, A Taroni - Rapporto ENEA RT/TIB, 1988

[21] J.F. Artaud et al 2010 Nucl. Fusion 50043001

[22] J. Candy et al., Physics of Plasmas 16, 060704 (2009)

[23] M. Barnes et al., Physics of Plasmas 17, 056109 (2010)

[24] M. Siccinio et al 2018 Nucl. Fusion 58016032

[25] A Kallenbach et al 2016 Plasma Phys. Control. Fusion 58045013

[26] D. Reiter et al. NF 30, 2141 (1990)

[27] H.-S. Bosch et al., PPCF 39, 1771 (1997)

[28] T. Puetterich et al., Impurity Limits in a Reactor Grade Fusion Device, 42nd EPS Conference on Plasma Physics P4.111 (2015) 
[29] L.E. Zakharov, in Plasma Physics and Controlled Nuclear Fusion Research, 1978 (international Atomic Energy Agency, Vienna, 1979), Vol. I, p. 689.

[30] Martin Y.R., Takizuka T. and The ITPA CDBM H-mode Threshold Database Working Group 2008 J. Phys.: Conf. Ser. 123012033

[31] C Angioni et al 2009 Plasma Phys. Control. Fusion 51124017

[32] S. Saarelma, private communication (2017)

[33] D. E. Post et al., ATOMIC DATA AND NUCLEAR DATA TABLES 20, 397-439 (1977) 